\title{
Le régime foncier camerounais face à l'exigence constitutionnelle de préservation des droits des populations autochtones
}

\author{
Par Prosper Nkou Mvondo
}

\section{Introduction}

La mise en place d'une nouvelle constitution ne peut laisser indifférent. Elle introduit des changements et l'on essaye de les présenter. Des bouleversements sont attendus dans l'ordre juridique et on peut déjà s'y interroger.

Depuis le 18 janvier 1996, un nouveau texte constitutionnel est entré en vigueur au Cameroun. ${ }^{1}$ Cette constitution, à travers les réformes qu'elle apporte, semble être la réponse que le pouvoir politique apporte aux revendications populaires qui ont plongé le Cameroun dans une crise sans précédent, où aucun secteur de la vie nationale n'aura été épargné dès le début des années $1990 .^{2}$ On entend, à travers cette constitution, instaurer des principes qui participent à plus de libéralisme dans un contexte de démocratie. On entend aussi protéger et préserver les droits des uns et des autres.

Portalis le disait si bien: «Le législateur ne doit pas perdre de vue que les lois sont faites pour les hommes, et non les hommes pour les lois, qu'elles doivent être adaptées aux caractères, aux habitudes, à la situation du peuple pour lequel elles sont faites.» ${ }^{3} \mathrm{Si}$ l'on a souvent reproché au législateur africain de ne pas faire preuve d'originalité ${ }^{4}$, on ne saurait

1 Pour les pouvoirs publics, il s'agit d'une révision constitutionnelle. En effet, il s'agit bien de la loi $\mathrm{n}^{0}$ 96/06 du 18 janvier 1996, portant révision de la Constitution du 2 juin 1972. Pourtant, Maurice Kamto estime qu'on est en présence d'une constitution nouvelle plutôt qu'une simple révision de la Constitution de 1972. Voir «Révision constitutionnelle ou écriture d'une nouvelle Constitution», Lex Lata, $\mathrm{n}^{0}$ 023-24, Févriér-Mars 1996, pp. 17 et s. Sur le processus d'élaboration de cette constitution, voir F. Mbome, Lex Lata, $\mathrm{n}^{\circ}$ 30, Septembre 1996, pp. 3 et s.

Les secousses politiques et l'effervescence populaire de 1990 ont d'abord amené le pouvoir politique à retoucher la constitution à travers la loi nº 91/001 du 23 Avril 1991 qui va réinstituer le poste du Premier Ministre. Discours préliminaire prononcé à la présentation du projet du Code civil, an XI.

4

La plupart des textes africains ne sont souvent que des reproductions quasi-conformes des textes européens. Des études ont été faites à propos J. Hilaire, La modernisation du droit dans les nouveaux Etats, in: Travaux de Recherches de l'Institut de Droit Comparé de Paris, Tome 30, 1965-66; voir E. Le Roy, L'introduction du modèle européen de l'Etat en Afrique francophone: 
nier au constituant camerounais du 18 janvier 1996 le mérite d'avoir eu en l'esprit cette pensée de l'illustre auteur.

Certes, on retrouve dans la Constitution du le 18 janvier 1996, la consécration de nombreux droits classiques qui figurent dans l'arsénal juridique de tout Etat qui se veut moderne: égalité de droits des citoyens, liberté et sécurité, inviolabilité du domicile, droits à la vie et à l'intégrité physique... Mais on y trouve aussi «des droits plus ou moins originaux qui expriment la spécificité camerounaise» ${ }^{5}$.

Les rédacteurs de la Constitution n'ont pas perdu de vue que le Cameroun est une mosaïque de tribus et d'ethnies ${ }^{6}$, les unes plus visibles sociologiquement que d'autres, les unes plus puissantes économiquement que d'autres... Et dans un contexte africain où nul, sauf mauvaise foi, ne pourrait nier la force des réalités tribales et ethniques, le constituant camerounais a compris qu'il fallait cesser de faire la «politique de l'autruche».

Aux revendications fédéralistes ${ }^{7}$, qui avaient pour prétention de résoudre à la fois les problèmes tribaux, ethniques, et de développement harmonieux du pays, le constituant oppose l'institution des régions dans un Cameroun unitaire. ${ }^{8}$ Aux revendications sousjacentes de certains autochtones, qui se sont toujours plaints de l'envahissement et de la violation de leurs droits ancestraux par des allogènes ${ }^{9}$, le constituant a su mettre le couteau

logique et mythologie du discours juridique, in: Décolonisation et Nouvelle Dépendance, sous la direction de C. Coquery-Vidrovitch et A. Forest, Presses Universitaires de Lille, 1984, pp. 81, 122; J. Foyer, Les destinées du droit français en Afrique, Penant, 1961, p. 1; G. Conac, Les Constitutions africaines et leur effectivité, in: Dynamique et finalités des droits africains, Economica, 1980. Ceci apparait souvent à travers des études comparées, voir Dagui Ngabo, La parenté en droit privé. Etude comparative du droit français et du droit post-colonial de l'Afrique noir, Thèse Droit, Poitiers, 1985.

J. Monangue Kobila, Le préambule du texte constitutionnel du 18 janvier 1996. De l'enseigne

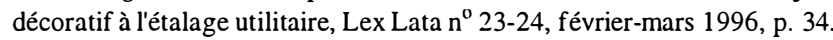

6 Le Cameroun est composé de près de 200 ethnies, QUID 1993, p. 914.

7

Ces revendications sont orchestrées par les dirigeants de la «All Anglophone Conference» qui dénoncent la marginalisation de la culture anglophone depuis le plébiscite du 11 février 1961 qui avait permis aux deux Etats du Cameroun oriental et du Cameroun Occidental de signer la Réunification du ler Octobre 1961. Jusqu'en 1972, le Cameroun est un Etat fédéral. C'est donc à un retour à la situation politique d'avant 1972 que prônait le «All Anglophone Conference». Voir art. 55 à 62 de la Constitution du 18 janvier 1996.

9 On peut lire dans l'Effort Camerounais de Janvier 1991, des revendications foncières sous la plume d'un prétendu autochtone de Yaoundé, l'abbé Abega Prosper. Le titre de l'article constitue en lui tout seul, un chef d'accusation: «L'urbanisation à Yaoundé: les autochtones en font les frais...». Dans l'article, on découvre des termes comme les «envahisseurs» (pour désigner les Camerounais allogènes), «la terre de nos ancêtres» (pour proclamer la légitimité des autochtones sur les terres de la capitale). Dans Le Patriote du 11/17 janvier 1993, on retrouve sous la plume de Ava Jean, Ewondo-autochtone de Yaoundé qui dénonce: «Les populations locales deviennent 
dans la plaie en juridicisant la notion sociologiquement connue d'autochtone ${ }^{10}$; et l'Etat s'engage à préserver les droits de ces autochtones.

On pourrait, sous plusieurs angles, s'interroger sur la portée de la notion constitutionnelle d'autochtone; ${ }^{12}$ mais on ne saurait perdre de vue que cette notion est étroitement liée à la terre. En effet, l'autochtone se définit comme celui qui est originaire de l'espace territorial où il habite. Lorsque la Constitution parle de «préservation des droits des populations autochtones», on comprend qu'il s'agit avant tout d'un droit à la vie et à la survie qui passe par un droit (ne serait-ce que naturel) sur la terre. Le Cameroun est un pays essentiellement agricole. Les populations en général et les autochtones en particulier, puisqu'il s'agit de ces derniers, ont avant tout besoin des terres pour cultiver ou faire de l'élevage, activités sans lesquelles il leur est difficile de vivre et de faire vivre leurs familles. Il y a donc forcément à travers le principe constitutionnel de préservation des droits des populations autochtones un enjeu foncier.

La finalité des textes de droit en Afrique noire en général a toujours été celle du développement. ${ }^{13}$ Au Cameroun en particulier, les textes de droit ont été orientés vers la construction nationale, le développement économique, social et culturel; des ambitions qui passent par la paix et l'unité nationale.

C'est dans cet esprit que la Constitution du 2 juin 1972 a été élaborée. Mettant fin au fédéralisme institué par la Constitution du ler septembre 1961, la Constitution de 1972 entend parfaire l'unité nationale. On institue la «République Unie du Cameroun», puis la «République du Cameroun» en 1984. Sous ce régime politique, tout est mis en oeuvre pour favo-

serfs. Cette logique aboutit à la situation dans laquelle les Ewondos se meuvent actuellement, sans espoir de sortir un jour. Le brassage des populations est souhaitable en soi (...). Mais là où le bât blesse, c'est lorsque nous constatons que dans la ville de Yaoundé et ses environs, les autochtones n'ont plus de terre, la terre de leurs ancêtres».

Voir Préambule de la Constitution: «L'Etat assure la protection des minorités et préserve les droits des populations autochtones conformément à la loi».

11 Il y a là matière à donner raison à Lavroff et Peiser qui écrivent: «On peut constater (...) dans les constitutions africaines la reconnaissance de certains droits originaux qui démontrent bien à quels problèmes les Etats africains ont à faire face», Lavroff / Peiser, Les Constitutions Africaines, Tome 1, Paris, Pedone, 1961, p. 19.

Juste après la promulgation de la Constitution, des intellectuels se sont lancés dans des réflexions sur la portée des notions constitutionnelles de minorité, d'allogène et d'autochtone. Voir Dossier La Nouvelle Expression-Documents, ${ }^{\circ} 1$ spécial du 12 Mai 1996, avec les contribution de RogerGabriel Nlep, Ngijol Ngijol, Sindjoun Pokam, Ktto Essome et bien d'autres.

Voir $K$. Mbaye, Droit et développement en Afrique francophone de l'Ouest, Revue Sénégalaise de Droit, $\mathrm{n}^{0} 1,1967$, pp. 23 et s.; J.Y. Toe, Le développement du droit au Burkina Faso, ou le lien dialectique entre droit et économie, Revue Burkinabé de Droit, $\mathrm{n}^{\circ} 11,1987$, pp. 273 et s. 
riser l'intégration nationale. On ne parle ni d'autochtone, ni d'allogène. Tous les Camerounais doivent se sentir égaux et protégés pareillement par les mêmes lois.

Faisant écho à la Constitution de 1972, la réforme foncière de $1974^{14}$ avait instauré des règles favorables à l'unité et à l'intégration nationale. C'est ainsi qu'il est admis que tout Camerounais puisse s'installer et investir partout où il le désire, à l'intérieur des frontières nationales. On n'a des comptes à rendre qu'à l'Etat, gestionnaire et distributeur des terres, des vastes étendues libres de toute occupation, que l'on appelle désormais «nal». La réalité autochtone est ignorée par la réforme de 1974. On accorde les droits à tous les citoyens sans distinction, ni discrimination.

La mise en application concrète de la politique foncière issue de la réforme a toujours soulevé des contestations. Elle a été l'objet de résistances de la part des autochtones qui ont peur de voir leurs terres ancestrales envahies par des «étrangers» ${ }^{15}$. En tout cas, les autochtones, possesseurs ou non des terres ${ }^{16}$, s'opposent à l'application du droit foncier étatique. Au delà des problèmes généraux d'ineffectivité des textes de droit en Afrique ${ }^{17}$, le droit foncier camerounais va apparaître comme source de conflits, parfois armés entre autochtones et «étrangers» ${ }^{18}$, mettant en péril la paix, l'unité et l'intégration nationales.

Un constat s'impose aujourd'hui: La réforme de 1974 connaît un échec sur le plan de son effectivité. Seulement, au-delà du droit foncier, c'est toute la politique de construction

14

15

16

17

A. Boni, La mise en pratique des lois dans les pays en voie de développement, Penant, 1963, p. 449; G. Conac, Les Constitutions africaines et leur effectivité, in: Dynamiques et finalités des droits africains, Economica, 1980; Bianc-X. Jouvan, La résistance du droit africain à la modernisation, Colloque de Dakar, Juillet 1977, Revue Sénégalaise de Droit, $\mathrm{n}^{\circ} 21,1977$, pp. 21 à 44; P.F. Conidec, Problèmes d'application de la législation en Afrique noire, in: Bulletin de l'IIAP, Avril 1977; Larba Yarga, De l'application des textes législatifs et réglementaires en Haute-Volta, in: Revue Voltaïque de Droit, $\mathrm{n}^{\circ} 1,1982$.

18

On peut lire dans Cameroon Tribune, $\mathrm{n}^{\circ}$ spécial du 5 janvier 1981, page 2, Affaire Mentchum: «Des disputes d'ordre domanial et foncier opposant agriculteurs et éleveurs dans le département de la Mentchum depuis le 27 décembre 1980, ont le ler janvier 1981, dégénéré en affrontements violents et en destruction de biens. Les efforts de modération et d'appel au calme des autorités administratives, traditionnelles et du parti étant demeurés vains devant l'extension des désordres, les forces de sécurité on reçu mission de rétablir l'ordre. Le bilan de ces incidents s'établit à sept morts, plusieurs blessés et de nombreuses cases incendiées»; Cité par A.D. Tjouen, Droits domaniaux et techniques foncières en droit camerounais, Economica, 1982, pp. 147-148. 
nationale mise en place depuis l'indépendance du pays qui a du mal à faire face aux réalités de tous les jours.

Les pouvoirs publics n'en sont pour autant pas désemparés. Ils gardent leurs ambitions de paix, d'unité et de développement. Il est seulement question pour eux, ayant tiré les conséquences de l'échec de l'ancienne stratégie, d'en mettre sur pied une nouvelle. Pour cela, il faut partir sur des bases juridiques neuves. La Constitution du le 18 janvier 1996 semble en être la concrétisation.

Pour étouffer les conflits entre autochtones et allogènes, la constitution tranche en faveur des autochtones dont elle entend préserver des droits particuliers. Mais de quels droits s'agit-il? Les autochtones ont-ils des droits propres dans l'ordre juridique camerounais? Si ces droits n'existent pas, sûrement que les textes législatifs et réglementaires ultérieurs en créeront, question d'être en conformité avec l'exigence constitutionnelle de préservation des droits des populations autochtones, préservation qui passe forcément par l'existence même de ces droits, il en existera sans doute plusieurs. Mais nous l'avons dit plus haut, la terre est le premier droit d'une population autochtone. C'est pour cela qu'il est intéressant de lancer d'abord le débat sur le droit foncier.

Les autochtones, qui sont désormais reconnus dans l'ordre juridique camerounais, ont-ils des droits fonciers spécifiques? S'ils n'en n'ont pas, on comprend alors que la réforme du régime foncier camerounais est une nécessité (I). Cette révision qui sera en quelque sorte une redistribution des terres, pourra-t-elle se faire sans que soit porté atteinte à des droits acquis? Il s'agira donc d'envisager les difficultés réelles à dégager un espace foncier propre aux populations autochtones (II).

\section{La nécessité d'une réforme foncière en faveur des populations autochtones}

La nécessité d'une réforme foncière au Cameroun se présente d'abord parce que le régime actuel, comme on peut l'observer, a du mal à s'appliquer sur le terrain. Cette absence d'effectivité étant liée entre autres, aux résistances que les autochtones ont toujours opposées, dénonçant ainsi la non prise en compte de leurs revendications dans la répartition des terres. C'est donc une nécessité d'ordre socio-juridique (A). La nécessité d'une réforme se présente ensuite parce que, quoiqu'il en soit, le texte législatif foncier doit se conformer à la constitution qui exige aujourd'hui la reconnaissance des populations autochtones et la préservation de leurs droits. Le texte foncier actuel, qui ne satisfait pas à ces exigences, est en contradiction avec la loi fondamentale. La reforme est alors une nécessité sur le plan strictement juridique (B). 


\section{A. Une nécessité d'ordre socio-juridique}

La répartition étatique des terres a toujours ignoré les revendications des populations autochtones (1); ce qui explique les résistances que ces populations ont toujours opposées au droit officiel (2).

\section{La répartition des terres à travers les différentes législations}

Le législateur post-colonial (b) adoptera la même politique que son prédécesseur colonial (a).

\section{a) La législation coloniale}

Après avoir obtenu des droits de souveraineté, de législation et d'administration du territoire camerounais en $1884^{19}$, les Allemands vont tout de suite effectuer une première répartition des terres. Ils reconnaissent aux autochtones des droits de propriété uniquement sur les emplacements sur lesquels se trouvent leurs villages et plantations. ${ }^{20}$ Le reste du territoire est considéré par la législation allemande comme «terres vacantes et sans maître» ${ }^{21}$. En réalité, ces terres seront versées au Domaine Impérial et c'est l'Etat allemand qui se charge de leur gestion. La distribution des «terres vacantes et sans maître» se fera quasi-exclusivement au profit des entreprises agricoles et forestières allemandes. ${ }^{22}$

Les autochtones camerounais contestent fortement cette répartition. Ils estiment que la conception de «terres vacantes et sans maître» n'est que la négation de leurs droits sur ces terres. Pour les autochtones, les terres non occupées appartiennent aux ancêtres et à leurs lignages. En effet, pour l'Africain, «affirmer que la brousse (...) n'appartient à personne est contraire à toute la tradition. Le coin le plus reculé de la brousse est sous la juridiction d'un chef quelconque, les autochtones ne peuvent admettre qu'une terre n'ait aucun rapport avec un groupe humain» 23 .

19

Traité Germano-Douala du 12 juillet 1884, signé entre E. Woerman et le Roi Akwa en présence de 23 témoins.

20

21

22

23

Art. 3 du Traité Germano-Douala.

Ordonnance Impériale du 15 juin 1896.

La Société Molowe-Pflanzung $\left(50 \mathrm{~km}^{2}\right.$ ), la Gesellschaft Nord-West-Kamerun (4 450000 hectares), la Gesellschaft Süd-Kamerun (7 200000 hectares); voir Bayiha, E., Les collectivités coutumières dans la législation camerounaise, Mémoire Licence, Université de Yaoundé, 1976-77, pp. 4 et s.

Olowale, E., La nature des droits coutumiers africains, Présence Africaine, 1961, p. 193. 
Les revendications des autochtones auront peu d'écho auprès des autorités coloniales. L'Etat allemand exige des autochtones une preuve écrite de leurs droits sur les terres revendiquées. Cette preuve va être impossible à établir, le droit précolonial sur lequel semblent se fonder les autochtones étant un droit non écrit.

Après la Première guerre mondiale, l'Allemagne perd sa colonie du Cameroun. Le territoire est placé sous-mandat de la Société des Nations. La France et l'Angleterre sont chargés de son administration. Avec ce changement d'autorité, les autochtones camerounais pensent récupérer les terres qu'ils ont toujours réclamées aux Allemands. Ils vont devoir se désillusionner, car la politique foncière franco-anglaise ne sera pas différente de celle des Allemands, tout au moins quant à la répartition des terres.

Côté anglais, on distingue deux catégories de terre: d'une part, les «terres vacantes et sans maître» (autrefois incorporées au domaine impérial allemand), appelées désormais «freehold lands» et placées sous la gestion de l'Etat colonial anglais ${ }^{24}$; d'autres part, on a les «native lands», les terres occupées par les autochtones. ${ }^{25}$ C'est la même répartition des terres que l'on retrouve côté français: les terres occupées par les autochtones d'une part, ${ }^{26}$ les «terres vacantes et sans maître» d'autre part. ${ }^{27}$

Il faut dire que la répartition coloniale des terres a énormément affecté les autochtones. même si les terres qui leur on souvent été accordées étaient des superficies considérables, il s'agissait dans la plupart des cas, de terres peu fertiles, les meilleures étant généralement classées «sans maître» et attribuées aux colons européens. ${ }^{28}$ Toutefois, les autochtones, toujours convaincus qu'il y a là une injustice liée à la politique coloniale de domination,

Comme à l'époque allemande, ces terres sont attribuées en priorité aux étrangers. On leur accordait des Certificates of Occupancy, droit réel d'occupation pouvant aller jusqu'à 99 ans.

25

Les autochtones se voyaient délivrer des "customary rights of occupancy» qui n'étaient en réalité que de simple attestations d'occupation sans garantie juridique.

26

On institue des certificats administratifs d'occupation et plus tard on passe on système des livrets fonciers qui comme les «customary rights of occupancy» du côté anglais, ne font que constater une occupation de la terre par les autochtones.

27

Décret du 12 janvier 1938; voir Code et Lois du Cameroun, Tome 4: «Les terres vacantes et sans maître appartiennent au territoiren; sur ces terres l'Etat colonial institue le régime de l'immatriculation aboutissant à un titre de propriété: le titre foncier. Seuls les colons européens et certains Camerounais riches ou assimilés aux Européens pouvaient obtenir ce titre.

28

Voir Rapport de l'autorité mandataire pour l'année 1925, Archives de Londres 1925: «Le fait d'être relégués dans les réserves a incontestablement fait perdre aux Bakweri beaucoup d'intérêt qu'ils portaient à la vie (...) même si les terres qui leur ont été allouées semblent d'une superficie suffisante pour survenir à leurs besoins. Elles sont invariablement peu fertiles, car les meilleures terres ont été attribuées aux étrangers»; cité par A.D. Tjouen, Droits domaniaux et techniques foncières en droit camerounais, Economica, 1982, p. 31. 
gardent la conviction qu'un jour, ils récupéreront leurs terres ancestrales. Beaucoup d'espoirs seront ainsi placés dans la législation post-coloniale.

\section{b) La législation post-coloniale}

Après l'indépendance du Cameroun en 1960, les autochtones ont cru que leur légitimité foncière serait reconnue sur les terres qu'ils occupent effectivement. Ils ont pensé que du simple «droit d'occuper» qui leur était reconnu à l'époque coloniale, le législateur de l'indépendance allait leur accorder un droit de propriété pur et simple. Loin d'améliorer la situation juridique des autochtones, le Décret-Loi de $1963^{29}$ ne parle aucunement de «propriété». A travers le texte, on retrouve plutôt les termes tels que «détention», «possession» qui traduisent le précarté du lien que les autochtones ont avec les terres qu'ils occupent.

La déception sera beaucoup plus grande en ce qui concerne les terres autrefois déclarées «sans maîtres» que les autochtones ont toujours réclamées. Le législateur de 1963 érigera ces terres en «Patrimoine collectif national» ${ }^{30}$ placé sous la gestion de l'Etat. L'Ordonnance du 6 Juillet 1974, texte de base du régime foncier camerounais en vigueur, reconnaît trois catégories de terre:

- les terres appropriées (objet d'un titre foncier);

- les domaines public et privé des personnes morales de droit public;

- le domaine national.

On remarque que dans cette répartition, aucune catégorie n'est spécialement attribuée aux populations autochtones. Les terres que celles-ci occupent ou exploitent sont de la dépendance du domaine national. ${ }^{31}$ A tout moment, les autochtones peuvent être victimes d'un déguerpissement suite à l'incorporation des terres qu'ils occupent aux domaines public ou privé des personnes morales de droit public. Ils n'ont droit dans ce cas, qu'à une indemnité correspondant à la valeur des constructions ou des cultures que supportait le terrain. ${ }^{32}$ L'Etat peut d'ailleurs, compte tenu de l'urgence, disposer de ces terres qui font partie du domaine national, sans indemnisation préalable. ${ }^{33}$

Décret-Loi $n^{\circ}$ 63/2/Cameroun Oriental du 9 janvier 1963 fixant régime foncier et domanial du Cameroun oriental (Journal Officiel, 15 janvier 1963, p. 31).

30

Art. 26 et 27, titre V du Décret-Loi de 1963, voir Y. Jouhaud, La notion du domaine ou patrimoine collectif national dans les nouvelles législations du Sénégal et du Cameroun, Revue Juridique et Politique Indépendance et Coopération, $n^{\circ} 1,1966$.

31

Article 15 de l'Ordonnance $n^{0} 74 / 1$ du 6 juillet 1974, fixant régime foncier.

32

Article 23 du Décret $n^{\circ} 76 / 166$ du 27 avril 1976 fixant les modalités de gestion du domaine national.

33

Article 24 du Décret $n^{0} 76 / 166$ sus-cité. 
Comme on peut l'observer, la législation officielle fait très peu cas des droits et revendications des populations autochtones. Elle va alors se heurter aux résistances de celles-ci.

\section{Les résistances des autochtones à la législation foncière}

Ces résistances se manifestent de manière passive (a) et le plus souvent de manière active (b).

\section{a) Les résistances passives}

Les résistances passives se caractérisent par une indifférence des populations autochtones au droit de l'Etat. On ignore par exemple l'existence de quelque titre officiel de propriété que ce soit. Depuis l'époque coloniale, les autochtones avaient la possibilité juridique de passer de la simple possession des terres que leur reconnaissait le législateur à un droit réel immobilier plus sécurisant côté anglais ${ }^{34}$, ou simplement à un droit de propriété par la procédure d'immatriculation qui aboutit à la délivrance d'un titre foncier, côté français ${ }^{35}$. Pourtant, on verra très peu d'autochtones engager la procédure d'obtention de ces titres officiels qui leur auraient conféré des droits réels au regard de la législation de l'Etat colonial.

Cette passivité des autochtones pourrait s'expliquer par l'attitude de l'administration coloniale qui, en dépit de la législation, rendait difficile la mise en oeuvre de la procédure, ou refusait tout simplement les titres plus protecteurs aux quelques autochtones qui osaient en faire la demande. Les étrangers étaient plus favorisés dans le cadre de ces attributions foncières, l'administration estimant qu'ils étaient plus aptes à mettre les terres en valeur. L'attitude des populations autochtones pourrait aussi s'expliquer par le coût prohibitif d'accession au titre foncier dans la partie coloniale française. Seuls en effet les colons européens (uniques riches à l'époque) et quelques Camerounais assimilés aux Européens ${ }^{36}$ pouvaient s'offrir de luxe d'une procédure d'immatriculation.

34 Côté anglais, rien d'interdisait aux autochtones de demander des «Certificates of occupancy» lui leur garantissaient une occupation pouvant aller jusqu'à 99 ans. On a même assimilé les certificates of occupancy à des titres de propriété définitifs.

35 J. Chabas, De la transformation des droits fonciers coutumiers en droit de propriété», Annales Africaines, 1959; $R$. Pamela, La procédure d'immatriculation foncière en Afrique, Thèse Droit, Paris 1955.

36 Qui étaient d'ailleurs pour la plupart des allogènes. 
Toutefois, il faut reconnaître que l'explication déterminante de cet engouement mitigé des autochtones à la course vers les titres officiels de propriété est tirée de ce que ces derniers se considèrent déjà, en vertu du droit coutumier, comme propriétaires des terres qu'ils occupent. Ils ont un sentiment de légitimité et dans leurs esprits, ils ont déjà un droit: un titre de propriété qu'ils tiennent de leurs ancêtres. L'autochtone ne ressent donc pas le besoin de se prémunir d'un autre titre.

On comprend dès lors pourquoi, même lorsque le législateur post-colonial de 1974 a simplifié la procédure d'obtention du titre foncier tout en la rendant gratuite ${ }^{37}$, très peu d'autochtones engagent des procédures d'immatriculation pourtant encouragés par les pouvoirs publics. Seuls les allogènes, le plus souvent, demandent des titres fonciers sur les parcelles qu'ils occupent. ${ }^{38}$ Mais, ces derniers risquent alors de faire face à l'impétuosité des autochtones qui parfois, de manière active, manifestent leur résistance à la législation foncière.

\section{b) Les résistances actives}

Les résistances actives des populations autochtones à la législation officielle étatique commencent avec la répartition allemande des terres. En 1910, les autochtones Doualas, dirigés par leur chef Douala Manga Bell, s'opposent violemment à l'occupation, par les Allemands du plateau Joss considéré par l'Etat colonial comme «terre vacante et sans maître». En réaction à cette attitude des populations, Douala Manga Bell est pendu par les Allemands le 4 août 1913. Quatre jours plus tard, Martin-Paul Samba, un autre chefautochtone s'opposant à l'occupation des terres par les colons est pendu à Ebolowa. ${ }^{39} \mathrm{La}$ période coloniale est ainsi émaillée de nombreux actes de résistance à la répartition des terres que l'administration essaye d'étouffer au tant que faire se peut.

Après l'indépendance, on assiste à un rejet par les autochtones de la notion de domaine national. Dans l'esprit du législateur étatique, les forêts, les savanes, occupées ou non, dès lors qu'elles ne font pas l'objet d'un titre foncier, relèvent du domaine national. Les autochtones quant à eux, sont loin de comprendre les choses ainsi. Ils considèrent les forêts et savanes comme leur propriété qu'ils entendent défendre par tous les moyens, même les plus violents. Lorsque le «propriétaire» d'une forêt y trouve des pièges, il somme celui qui

37

38

39 
les a tendus de les enlever. La non-observation de cette injonction par «l'étranger-chasseur» amène ce «propriétaire» à les détruire. ${ }^{40}$

Quelquefois, c'est l'institution même du titre foncier qui est violemment attaquée par des autochtones. L'affaire Abessolo en est une illustration. Abessolo Hubert entreprend d'immatriculer un terrain et obtient un titre foncier régulier sur ladite parcelle. Il est au regard du droit de l'Etat, devenu définitivement propriétaire dudit lot. Investi de tous les attributs du droit de la propriété (usus, fructus, abusus), Abessolo Hubert s'engage alors à vendre, par morcellement, ledit terrain. Son frère Abessolo Luc, s'oppose à ces ventes, estimant qu'il s'agit là des terres relevant du patrimoine familial. C'est ainsi que chaque fois que des bornes sont of ficiellement placées par les services du cadastre, Abessolo Luc amène toute une équipe composée des membres de sa famille et des amis pour les déplanter et les jeter. Parfois même sur le terrain, avec son équipe, il engage des luttes avec les géomètres. ${ }^{41}$

On a également vu en 1993 dans un quartier de Yaoundé, des autochtones constitués en milices pour empêcher des légitimes propriétaires, titulaires de titre foncier, de viabiliser leurs biens immobiliers. ${ }^{42}$ Il est clair aujourd'hui que, l'arrivant qui entend se prévaloir du droit de l'Etat et de son titre foncier pour s'installer sur des terres revendiquées par des autochtones, s'expose à des agressions de la part de ces derniers. Les allogènes camerounais l'on compris et, par leur attitude, ils semblent reconnaître la légitimité foncière des autochtones sur les terres. On verra ainsi le chasseur-allogène déguerpir de lui même, après sommation de l'autochtone qui se dit «propriétaire» de la forêt vierge en cause, sans chercher à se plaindre devant quelque autorité que ce soit. Le chasseur semble être conscient d'avoir empiété sur les terres d'autrui.

Pour s'assurer une installation paisible, le nouvel arrivant préfere «négocier» avec l'autochtone qu'il trouve sur place. Au lieu de passer par une procédure d'immatriculation qui lui garantit une protection juridique étatique, l'allogène rencontre l'autochtone «propriếtaire». Dans un contrat sous-seing privé, l'arrivant s'engage en échange d'une parcelle, à verser une somme d'argent. Il s'oblige aussi tacitement ou non à fournir des prestations secondaires relevant plus d'un rituel religieux: fourniture de vin, d'une chèvre, d'un pagne... Pour caractériser ce type de contrat que l'on retrouve aussi en Côte d'Ivoire, A. Fian utilise la notion de «précarité»" ${ }^{43}$. Dans l'esprit de l'autochtone, il n'y a pas cession de propriété. Il y a simplement cession d'un droit d'usage précaire et révocable. L'autochtone reste «pro-

40

41

42

43

Voir A.D. Tjouen, Droits domaniaux et techniques foncières (déjà cité, note 18), p. 62.

Voir A.D. Tjouen (déjà cité, note 18), p. 95.

Voir La Nouvelle Expression, nº 88 du 24/30 août 1993.

A. Fian, Le droit foncier de l'Etat Ivoirien, Thèse Droit, Paris 1992, p. 365. 
priétaire». Il demeure rattaché à la parcelle, laquelle est le trait d'union qui le maintient en contact avec ses ancêtres.

Les pouvoirs publics semblent aussi reconnaître la légitimité foncière des autochtones, jouant ainsi le jeu de ceux-ci au détriment du droit officiel. On verra ainsi qu'après l'activité des milices dont il a été fait état dans un quartier de Yaoundé, et sous la pression des autochtones, des titres fonciers régulièrement délivrés à des allogènes ont été purement et simplement retirés ${ }^{44}$, et les terres rétrocédées aux populations autochtones ${ }^{45}$, remettant ainsi en cause le principe d'intangibilité du titre foncier. On a alors parlé d'attribution d'un «espace vital vivrier» à la communauté villageoise de Ngousso. ${ }^{46} \mathrm{Si}$ on peut admettre que de telles décisions soient prise dans un souci de préserver la paix sociale, il faut aussi reconnaître qu'il s'agit là de décisions au fondement juridique discutable.

Les résistances, et la manière dont celles-ci sont gérées, montrent que tous les intervenants dans les problèmes fonciers semblent admettre une légitimité des autochtones sur les terres que ces derniers occupent. Seule la législation foncière est en marge de ce mouvement de légitimation. Cette législation devrait donc être repensée pour être en conformité avec les réalités et surtout pour les besoins de paix sociale; une paix qui passe forcément par la reconnaissance des droits fonciers aux populations autochtones. L'existence effective des résistances a perınis aux pouvoirs publics de comprendre que les autochtones existent et qu'ils ne veulent pas voir leurs droits sur la terre ignorés.

En 1993, en plein coeur des revendications politiques, un «large débat» national est ouvert en vue de la rédaction d'une nouvelle constitution. Les aspirations populaires devraient y être prises en compte. Les autochtones en ont alors profité pour faire part de leurs inquiétudes d'abord quant à leur existence et ensuite quant à leurs droits. Le 18 janvier 1996, le texte constitutionnel est promulgué et on y retrouve le principe de «préservation des droits des populations autochtones». La législation foncière doit donc trouver à travers cette disposition constitutionnelle, la base juridique d'une réforme.

\section{B. Une nécessité d'ordre purement juridique}

Le principe de préservation des droits des populations autochtones est fixé par le préambule et sa constitutionnalité ne souffre pas de doute (1). La supériorité de la constitution dans la

Arêté $n^{\circ}$ 170/Y/2/6/MINUH/D210 du 8 juin 1993 remettant dans »leurs droits réels de propriété, les anciens attributaires du lotissement de Ngousso".

Arrêté $n^{\circ}$ 171/Y/2/6/MNUH/D210 du 8 juin 1993 consolidant les droits réels de propriété des populations «originaires» de Ngousso.

Voir La Nouvelle Expression (dé jà cité, note 42). 
hiérarchie des normes juridiques doit pouvoir contraindre les lois foncières à s'y conformer (2).

\section{La constitutionnalité du principe de préservation des droits des populations autochtones}

Le principe apparaît un peu trop abstrait. On peut s'interroger sur son effectivité et sur son réalisme. Mais il faut d'abord se poser des questions sur sa juridicité. S'agit-il d'une norme revêtant un caractère obligatoire? Le problème se pose parce que le principe figure dans le préambule de la constitution. Il n'y a pas de doute sur la juridicité d'une disposition constitutionnelle lorsque celle-ci se trouve dans le dispositif même de la constitution. Des auteurs ont souvent estimé que cette juridicité était moins sûre lorsque la norme figure seulement dans le préambule.

La question sur la valeur juridique du préambule a donné lieu à une controverse doctrinale ${ }^{47}$ où deux opinions contraires ont été soutenues. Pour des auteurs comme Carré de Malberg, Esmer, Leferrière, les déclarations des droits contenus dans le préambule ne seraient que des énoncés de principes philosophiques et moraux sans valeur juridique. Ils s'appuient sur le fait que les principes qui y sont énoncés le sont en termes très généraux, très vagues. Une thèse contraire, défendue par Hariou et Duché, soutient que le texte du préambule a la même valeur juridique que les dispositions de la constitution stricto sensus. Toutefois, ce courant doctrinal exige que les droits énoncés dans le préambule soient précis et susceptibles d'application pratique.

La controverse n'a jamais été que doctrinale. Elle a été aussi jurisprudentielle. En France, le Conseil d'Etat ${ }^{48}$ a refusé toute «valeur positive et constitutionnelle» au préambule de la Constitution du 27 octobre 1946. Dans un arrêt de 1957, le Conseil d'Etat reconnaît une valeur juridique au préambule de la même Constitution. Il va falloir attendre l'arrêt du

Sur la controverse, on peut voir $F$. Batailler, Le Conseil d'Etat, Juge constitutionnel, Paris, Librairie Générale de Droit et de Jurisprudence, 1966; G. Vedel, Droit administratif, Sè édition, Paris, Presses Universitaires de France, 1973; F. Luchaire, Le Conseil constitutionnel, Paris, Economica, 1980; C.I. Franck, Les fonctions juridictionnelles du Conseil constitutionnel et du Conseil d'Etat dans l'ordre constitutionnel, Paris, Librairie Générale de Droit et de Jurisprudence, 1974; D. Turpin, Contentieux constitutionnel, Paris, Presses Universitaires de France, 1986; S.A. Ould Bouboutt, L'apport du Conseil constitutionnel au droit administratif, Paris, Economica, 1987.

CE, 7 juillet 1950 (Arrêt Dehaene), Revue de Droit Public, 1950, p. 691, conclusions; Grazier, Arrêt Tessier, Dalloz, 1953, p. 735, conclusions; Donnedieu de Vabres; voir aussi C. Franck, Les grandes décisions de la Jurisprudence - Droit constitutionnel, Paris, Presses Universitaires de France, 1978, pp. 18 et s.; Louis Favoreu / Loic Philip, Les grandes décisions du Conseil constitutionnel, 4e édition, Paris, Sirey, 1986, p. 249. 
Conseil d'Etat du 17 février 1960 (Société EKY), pour voir le Conseil d'Etat prendre nettement position en faveur d'une reconnaissance de la valeur juridique du préambule de la constitution du 4 octobre 1958. ${ }^{49}$ Aujourd'hui, le débat est clos en France avec la décision du Conseil Constitutionnel du 16 juillet $1971 .^{50}$ La discussion est restée ouverte, s'agissant de la valeur juridique du préambule de la plupart des constitutions africaines. ${ }^{51}$

A propos du Cameroun, alors que Eric Boehler reconnaît une valeur juridique certaine au préambule de la Constitution de 1961 et $1972^{52}$, Pougoue et Kamto contestent toute valeur juridique au préambule de la Constitution de $1972 .^{53}$

Le texte constitutionnel du 18 janvier 1996 vient lui-même trancher le débat en consacrant expressément la valeur juridique de son préambule. Plus d'ambiguité, plus de discussion à propos: l'art. 65 énonce clairement que «le préambule fait partie intégrale de la Constitutions 54 .

On comprend alors que les principes posés par le préambule de la Constitution du 18 janvier 1996, entre autres, celui de la «préservation des droits des populations autochtones» ne sont pas que de simples principes philosophiques et moraux exempts de toute valeur juridique. Ces principes sont sources du droit positif et sont pourvus d'une force contraignante incontestable. Le droit foncier ne peut que s'y conformer.

CE, 17 février 1960, Société Eky, Sirey, 1960, conclusions; Kahn, Juris-Classeur Périodique, 1960, II. 10629 (bis), note Vedel.

50

Décision Liberté d'Association, 16 juillet 1971, Recueil des décisions du Conseil Constitutionnel, p. 29, Revue de Droit Public, 1171, note J. Robert, Dalloz, 1974, 83, Chronique, L. Hamon.

51 Voir M. Kamto, L'énoncé des droits dans les Constitutions des Etats africains, Revue Juridique Africaine, $n^{\circ} 2$ et 3,1991, p. 13.

52

Voir Arrêt n 178 du 29 mars 1972, Mouelle Koula Eitel contre/ République Fédérale du Cameroun; Arrêt nº 194 du 25 mai 1972, Nana Tchana Daniel contre/ République Fédérale du Cameroun, in: Revue Camerounaise de Droit, $n^{0} 3$, janvier-décembre 1973, p. 54; obervation Eric Boehler.

Voir Pougoue / Kamto, Commentaire de la loi $n^{0} 89 / 018$ du 28 juillet 1989 portant modification de la loi $n^{0} 75 / 16$ du 08 décembre 1975 fixant procédure et fonctionnement de la Cour suprême, in: Juridis Info $\mathrm{n}^{0} 1$, janvier-février-mars 1990, p. 8.

Le constituant camerounais s'est sans doute inspiré de certaines constitutions africaines où le débat a été clos de manière similaire. Ainsi, la Constitution de Haute-Volta du 29 juin 1970 précisait au paragraphe VIII de son préambule: «Les dispositions qui précèdent (celle du préambule) font partie intégrale de la présente Constitution». La Constitution du 27 novembre 1977 indiquait plus clairement encore: «Le présent préambule est partie intégrante de la Constitution» (paragraphe XXI du préambule). Gonidec reconnaît qu'une telle prise de position reste fort rare, Les droits Africains. Evolutions et sources, 2è Edition, Paris, Librairie Générale de Droit et de Jurisprudence, 1976, p. 101. 
2. La suprématie du principe constitutionnel de préservation des droits des populations autochtones sur les lois foncières

«La constitution domine le législateur». Il faut dire que pendant longtemps, les juristes français n'ont pas fait cas de cette pensée de Alexis de Tocqueville. Ils considéraient la Constitution comme une simple charte politique, codifiant les rapports entre les différents organes de l'Etat. L'irruption du droit constitutionnel sans les autres branches du droit ne pouvant qu'être exceptionnelle et largement marginal. Par conséquent, le législateur, en matière de droit privé notamment, n'est pas tenu de se soumettre aux exigences constitutionnelles. «Le dogme de la souveraineté parlementaire, au nom duquel le législateur, parce qu'il ne peut jamais mal faire, peut tout faire, a transformé la constitution, norme suprême en une simple déclaration de volonté qui doit inspirer les majorités parlementaires, sans jamais toutefois les lier», écrit Marc Frangi ${ }^{55}$. Pour expliquer cet effacement du droit constitutionnel par rapport aux autres droits, l'auteur évoque l'absence dans la France de l'époque, d'un véritable contrôle de constitutionnalité.

L'absence d'un véritable contrôle de constitutionnalité des lois ${ }^{56}$ expliquerait sans aucun doute la liberté que le législateur camerounais s'est souvent donnée avec les principes constitutionnels. Dans un contexte où la constitution connaît toutes les infortunes dans la pratique $^{57}$, et ou elle ne revêt aucune signification profonde parce qu'elle manque d'enracinement historique; dans un contexte où, comme le dit Maurice Kamto, les Constitutions et les déclarations qui les ornent semblent décrire un modèle idéal de société dont les auteurs eux-mêmes sont d'avance persuadés qu'il est irréalisable ${ }^{58}$, la constitution camerounaise a souvent été bafouée par des lois et des actes réglementaires de circonstance. On citera a cet effet, les Ordonnances du 28 septembre 1972 qui violent ostentoirement le principe constitutionnel de la non-rétroactivité des lois 59 .

La question de la constitutionnalité de l'ensemble des branches du droit ne se pose plus dans les Etats modernes. En France, les multiples atteintes aux droits fondamentaux durant l'occupation ayant montré la nécessité de mieux assurer la protection des droits et libertés

55

M. Frangi, L'apport du droit constitutionnel aux droits des personnes et aux droits économiques individuels. Contribution à l'étude de la constitutionnalité du Droit privé, Thèse Droit, Aix Marseille III, 1990, p. 6.

56

57

58

59

Voir F. Mbome, Le Contrôle de la Constitutionnalité des lois au Cameroun.

Voir $G$. Conac, Le Droit reconnu par les gouvernants, in: La vie du droit en Afrique, dans Dynamiques et Finalités des Droits Africains, Economica, 1980, p. XVIII; voir aussi Les constitutions africaines et leur effectivité, même ouvrage, pp. 385 et $\mathrm{s}$.

Voir M. Kamto (déjà cité, note 51), p. 21.

Voir $R$. Sokeng, Le visage inconstitutionnel du code pénal camerounais, Lex Lata n ${ }^{\circ} 29,1995$, pp. 3 et $s$. 
de l'Homme, la Constitution du 27 octobre 1946 a mis pour la première fois un organe indépendant chargé de veiller à la compatibilité des lois avec le texte constitutionnel. Tous les textes législatifs doivent se conformer aux exigences constitutionnelles. Le contrôle de constitutionnalité s'est énormément accru. La jurisprudence du Conseil constitutionnel a désormais une grande influence sur l'évolution de l'ensemble du droit ${ }^{60}$. La constitution est devenue la principale source du droit. C'est elle qui détermine les principes essentiels des diverses branches du droit. On doit admettre aujourd'hui avec Vedel et Dévolvé que «la constitution n'est pas seulement destinée à organiser les pouvoirs publics, fixer leurs compétences, régir leurs rapports, préciser leurs procédures: elle établit le cadre de la vie sociale, donc de la vie juridique, et par là même, doit comporter les normes de fond qui la régissent (...). On pourrait ainsi considérer que toute constitution constitue la base matérielle du système juridique» ${ }^{61}$.

Sur la base des développements qui précèdent, il est indéniable que la Constitution camerounaise du 18 janvier 1996 doit avoir une influence sur l'ensemble du droit, et par conséquence, sur le droit foncier. C'est la constitution qui définit donc les principes supérieurs qui gouvernent le droit foncier. On peut d'ores et déjà parler d'un droit constitutionnel foncier, lequel pose le principe de «préservation des droits des populations autochtones».

Le respect des principes constitutionnels s'impose au législateur ordinaire, ce dernier est tenu, comme le dit Mbendang Ebongue Job, d'obligations qui peuvent être positives ou négatives. 62

- Pour les obligations négatives, c'est clair que le législateur ne pourrait pas voter une loi en contradiction avec un principe constitutionnel. Qu'en est-il lorsqu'une loi précédant une constitution est en contradiction avec cette dernière? La question est réglée par l'art. 68 de la Constitution du 18 janvier 1996 qui dispose que la législation actuelle applicable reste en vigueur dans ses dispositions qui ne sont pas contraires à la Constitution. Peut-on dire qu'il y a des dispositions dans le régime foncier actuel en contradiction avec le principe constitutionnel de préservation des droits des populations autochtones? La réforme foncière de 1974 reconnaît à tous les Camerounais (et par conséquent aux autochtones), un droit d'occupation sur les espaces qu'ils occupent ou qu'ils exploitent. Mais ces droits ne sont nullement protégés. Existent des mesures prévues par le texte foncier qui peuvent s'avérer aujourd'hui inconstitutionnelles, par

Voir L. Favoreu, L'influence de la jurisprudence du Conseil Constitutionnel sur les diverses branches du Droit, in: Mélanges Léo Hamon, Economica, 1982.

Vedel / Devolve, La constitution comme base du système juridique, Revue Internationale de Droit Comparé, 1979, p. 122.

62

Mbendang Ebongue, Constitution et droit du travail: contribution à l'étude de la constitutionnalisation du droit privé en Afrique noire francophone: le cas du Cameroun, Revue Juridique Africaine, $n^{0} 1 / 2 / 3$, pp. 125 et $s$. 
le déguerpissement d'une population autochtone de l'espace qu'elle occupe, avec ou sans indemnité préalable.

- Pour les obligations positives, elles s'imposent au législateur selon Mbendang Ebongue, dans la mesure où les principes posés par la constitution ne sont pas encore réalisés par la législation ordinaire. Actuellement, cette obligation s'impose au législateur foncier. Ce dernier devra voter des lois en conséquence pour mettre les principes constitutionnels en oeuvre.

Aujourd'hui que la notion de «population autochtone» est entrée dans l'ordre juridique camerounais à travers le texte constitutionnel du 18 janvier 1996, le législateur se doit de mettre sur pied une réforme qui intégrera la notion dans le régime foncier. Cette réforme doit d'abord reconnaître des droits fonciers aux populations autochtones, car il n'y a point préservation de droits sans l'existence même de ces droits. Mais la tâche qui incombe désormais au législateur foncier n'est pas des plus faciles.

\section{Les difficultés juridiques d'une réforme foncière au profit des populations autochtones}

Le constituant n'a nullement défini la notion d'«autochtone». Il appartient au législateur d'apporter des précisions sur cette réalité sociologique devenue juridique (A). La loi devra aussi définir l'assiette des droits à accorder aux autochtones et dire comment les préser$\operatorname{ver}(\mathrm{B})$.

\section{A. Les difficultés d'une définition juridique de la notion de population autochtone}

Ces difficultés sont essentiellement de deux ordres: le législateur aura du mal à traduire juridiquement la notion d'autochtone (1). Si une définition est trouvée, il lui sera alors difficile de déterminer les personnes faisant partie d'une population reconnue comme autochtone (2).

\section{La difficile jurisdicisation d'une notion sociologique}

S'agissant des considérations sociologiques, il faut savoir que lorsqu'on parle de «populations» dans le contexte camerounais, il faut entendre des collectivités formées sur des bases 
tribales et ethniques; ce que l'on appelle encore «collectivité coutumière», un terme déjà connu en droit foncier camerounais.

Dans les campagnes, la population d'un village n'est généralement composée que des membres d'une grande famille lignagère rassemblés autour d'un chef coutumier. La collectivité coutumière et le village ne font alors qu'un. Dans les grandes agglomérations, on retrouve des milliers de collectivités coutumières. La ville est composée de plusieurs «villages artificiels». Le terme se rapporte ici à la reconstruction au sein des villes, de certaines réalités traditionnelles et villageoises. On assiste alors à un prolongement fictif du village en zone urbaine. ${ }^{64}$ Des quartiers ont ainsi spontanément vu le jour dans les grandes villes camerounaises, habités (parfois exclusivement) par des ressortissants d'une même ethnie ou d'une même tribu. Ces quartiers sont d'ailleurs désignés par l'origine de leurs habitants. A Douala ou à Yaoundé par exemple, on entendra parler de «quartier Bamileké», «quartier Bassa», «quartier Haoussa» etc. 65

Les habitants ainsi regroupés dans les villages et dans les centres urbains semblent correspondre à ce qu'on appelle: «populations». Le problème ici est celui de dire, quelles sont les «populations» que l'on doit considérer comme autochtones par rapport à un espace occupé en ville ou au village. Le terme «autochtone» se dit des populations originaires des pays qu'elles habitent, des populations qui ne sont pas venues par immigration. ${ }^{66}$ On pourrait donc aisément dire d'un individu qu'il est autochtone du Cameroun, dès lors qu'il y vit et qu'il est établi que ses ancêtres y ont toujours vécu.

Pourtant, dans la sociologie camerounaise, le concept d'autochtone n'est pas compris de la sorte. On retrouvera à l'intérieur des frontières du Cameroun, des citoyens de souche considérés dans un espace donné, comme «étrangers» par d'autres citoyens se disant «autochtones». Le phénomène est très visible lorsqu'il s'agit de l'occupation et de l'exploitation des terres. Le Doyen Melone a su l'appréhender: «Lorsqu'il s'agit d'un problème important comme celui du droit foncier, le concept d'étranger est plus précis. Est traité comme étranger celui qui n'a pas les mêmes coutumes, ne parle pas la même langue et qui n'est pas

Article 17 de l'Ordonnance $\mathrm{n}^{0} 74 / 1$ du 6 juillet 1974 à titre d'exemple.

Voir J.B. Fotsing, Pouvoir fiscal en Afrique: Résistance du système traditionnel au système modeme, Penant, 1990, p. 73.

Des chercheurs se sont intéressés au phénomène voir $J$. Merlo, La retribalisation moderne d'une grande ville d'Afrique noire: les Doualas et les Bassas à Douala, cité par P. Nassara, in: Aspect de domination politique au Cameroun, RFEPA $n^{\circ} 113$, mai 1973; B. Delpech, Du village au quartier: les originaires de la Lékié à Yaoundé (Nkol-Eton), Institut des Sciences Humaines, $\mathrm{n}^{0} 10$, Yaoundé, Onarest, 1978-

Voir la définition de Littré, Tome 1, p. 737. Lire aussi: Ngijol Ngijol, Autochtones, allogènes et minorités au Cameroun, La Nouvelle Expression (déjà cité, note 42), p. 2. 
originaire d'un groupe allié.» ${ }^{67}$ N'est donc seulement pas étranger le Français, le Gabonais ou le Chinois qui se retrouve sur le territoire camerounais. L'est également, dans l'esprit du Camerounais, son compatriote de la tribu Bamileké de la Région de l'Ouest, lorsqu'il se retrouve à Yaoundé, fief ancestral de la tribu Ewondo. Plus encore, dans un village, un Camerounais appartenant à un autre village est un «étranger» par opposition à celui qui a des souches ancestrales dans ledit village et que l'on nomme: «autochtone».

La politique gouvernementale depuis l'indépendance, jointe à la Constitution de 1972 et aux travaux préparatoires de la réforme foncière de 1974, ont toujours invité les Camerounais à la tolérance, en se détournant du terme «étranger» lorsqu'il s'agit d'autres Camerounais. Il est question de permettre à tout Camerounais de se sentir chez lui partout où il se trouve sur l'ensemble du pays. Cette politique d'intégration nationale a été approuvée en théorie par les populations. Sur le terrain, on observe toujours des résistances et des difficultés réelles d'intégration des populations «non-autochtones» lorsqu'il s'agit de l'occupation et de l'exploitation des terres. ${ }^{68}$ Il y a en tout cas dans la sociologie camerounaise, d'une part des populations «autochtones» et, d'autre part, des «étrangers» qu'un auteur invite à appeler «allogènes». ${ }^{69}$ Cette discrimination entre Camerounais, observable sur le plan sociologique, n'avait jusque l'a jamais été admise sur le plan juridique. Elle est désormais consacrée par la Constitution du 18 janvier 1996 qui reconnaît formellement l'existence des populations autochtones. Le terme est entré dans l'ordre juridique camerounais.

Un intérêt certain, juridiquement protégé est d'ores et déjà attaché à la qualité d'autochtone. Il importe donc de dire dès à présent qui, par rapport à une portion du territoire national, est autochtone ou ne l'est pas. En d'autres termes, il va falloir donner une définition juridique à la notion d'autochtone. Cette définition devra permettre de déterminer avec précision les populations considérées comme autochtones sur une parcelle foncière. Les difficultés sont énormes. Toutefois, des solutions peuvent être proposées.

Le législateur pourrait définir une «population autochtone» comme une collectivité coutumière installée sur une parcelle du territoire national, où elle n'est pas arrivée par immigration. Ce serait là reprendre simplement l'étymologie du terme autochtone. Malheureuse-

S. Melone, La parenté et la terre dans la stratégie de développement. Exemple du Sud Cameroun, Thèse Droit, 1968, p. 44.

68

Voir l'article de Ava, Jean in Le Patriote (déjà cité, note 9) pour comprendre l'ampleur du rejet des populations non-autochtones au Cameroun. L'auteur s'attaque particulièrement à une tribu qu'il trouve envahissante: «Les idéologues du fascisme occidental de notre pays nous font donc savoir que le Bamiléké est un être supérieur qui a droit à tous les égards et surtout qu'il peut s'installer partout au Cameroun en toute impunité (...). Les Bamilékés arrivent quelque part (...) sous le couvert de l'unité nationale, pour quémander du terrain (...). Alors commence l'exploitation éhontée de la terre des Ewondos.» 
ment, cette définition risque de n'avoir aucune correspondance dans la réalité camerounaise. En effet, quelques certitudes fondées sur des études ethnologiques et anthropologiques, permettent d'établir l'unanimité sur le fait que les populations camerounaises se sont essentiellement formées grâce à des flux migratoires. Celles qui occupent un espace aujourd'hui et qui se prétendent autochtones sont bien arrivées un jour et s'y sont installées $^{70}$; parfois en repoussant les occupants qu'elles ont trouvés sur place, peut-être véritables «autochtones» ${ }^{71}$. Il est aujourd'hui difficile de dire avec exactitude quelles sont les populations autochtones. Cette difficulté est liée à l'oralité de la littérature africaine qui n'a pu fixer l'histoire de manière précise.

La définition juridique d'une population autochtone devrait donc se détacher de l'étymologie du terme. Toute référence à une première installation sur la terre devrait être écartée. Pour Nlep, il faut restituer la définition d'autochtone par rapport à l'histoire propre du Cameroun. Selon ce juriste, «est autochtone (...) au plan des réalités historiques, sociologiques, géographiques, celui qui était installé quelque part en 1884. Il est autochtone de cet endroit» ${ }^{72}$. Cette définition semble lier la notion d'autochtone à la naissance de l'Etat camerounais, en même temps qu'elle légitime la première répartition des terres effectuée par l'Etat colonial allemand. En tout cas, Nlep a choisi une date marquante de l'histoire étatique et foncière du Cameroun comme point de départ de détermination du premier occupant foncier. En restant dans sa logique, on pourrait tout aussi bien choisir une autre date ayant une forte signification étatique et foncière. Pourquoi ne choisirait-on pas 1919, l'arrivée des Français et des Anglais? 1960, l'année de naissance de l'Etat post-colonial? 1974, la date de la dernière réforme foncière? Et pourquoi pas 1996, date d'entrée en vigueur de cette Constitution qui a fait entrer le concept de populations autochtones dans l'ordre juridique camerounais?

Quelle que soit la date butoir retenue, celle-ci ne sera que le reflet d'un arbitraire législatif. Et il faudra s'attendre à des contestations et revendications: ainsi, en retenant une date lointaine comme 1884, on trouvera sûrement des populations installées quelque part depuis plus d'un siècle qui se verront refuser la qualité d'autochtone. ${ }^{73} \mathrm{Il}$ y a fort à parier qu'elles ne l'admettront pas facilement. A l'inverse, en choisissant une date récente comme 1974, des populations installées seulement depuis vingt-cinq ans se verrons reconnaître la qualité

L'étude des migrations dans la zone de Yaoundé (la capitale) montre que les Bétis qui s'y trouvent aujourd'hui sont loin d'être les premiers occupants de cet espace foncier. Leur arrivée en ces lieux remonte à la fin du 18 è siècle et début du 19 è siècle.

Il est établi qu'à Douala, la tribu Douala qui occupe le sol de cette ville aujourd'hui, vient du BasCongo et que ce n'est que vers le 15 è siècle qu'elle s'installe après avoir repoussé les Bassas et Bakokos par des guerres tribales.

R.G. Nlep, Penser l'Afrique selon nos réalités, La Nouvelle Expression (déjà cité, note 42), p. 18.

Une population installée depuis 1890 par exemple, ne sera pas considérée comme autochtone. 
d'autochtone; ce qui sera fait sans doute pour mécontenter celles qui y sont depuis plus d'un siècle. Dans l'un et l'autre cas, il y a un risque d'assister à des résistances dans l'application d'une telle législation. La mission du législateur est sur ce point assez délicate. Peut-être finira-t-il par se décider; mais là ne s'arrêteront pas ses difficultés. Après avoir dit ce qu'est une population autochtone, le législateur devra encore dire quels sont les individus qui en font partie.

\section{Les difficultés liées à la détermination des personnes faisant partie d'une popula- tion autochtone}

La Constitution parle de «populations autochtones» dont l'Etat s'engage à préserver les droits, et non de «personnes» ou d'individus». Faut-il comprendre que les droits en question ne peuvent être revendiqués que collectivement? La protection des droits collectifs et leur exercice collectif ont pris de l'ampleur dans l'évolution récente du droit. On parle ainsi de protection des consommateurs, par exemple. Toutefois, l'existence d'un droit collectif n'interdit nullement à un individu isolé de le revendiquer et l'exercer. L'organisation collective n'est faite que pour mieux assurer la protection individuelle. On peut dont dire que le droit d'une population est aussi, et avant tout d'ailleurs, le droit d'un individu faisant partie de cette population.

Il ne faut pas perdre de vue que le Cameroun est en plein dans un système d'économie libéral; et qu'il y a longtemps que le collectivisme traditionnel africain a cédé la place à l'individualisme occidental. ${ }^{74}$ Relativement au foncier, le droit de la terre ne se conçoit plus collectivement; c'est chaque individu appartenant ou non à un groupe social, qui entend, pour ses besoins économiques propres, obtenir à titre privatif ${ }^{75}$, une portion individuelle de terre. Il faut donc s'attendre à voir des individus se prétendant autochtones, revendiquer la préservation constitutionnelle de leur droit. Il faudra alors que l'individu établisse qu'il fait partie d'une population reconnue comme autochtone. Il revient au législateur d'en fixer les règles.

On est loin de penser que le législateur mettra en place des critères morphologiques et culturels pour établir le lien entre un individu et une collectivité reconnue comme autochtone. Bien que la notion de population soit liée à la tribalité et à l'ethnicité, un tel procédé serait inconséquent pour plusieurs raisons: d'abord parce qu'il est difficile, voire impossible

On pourra lire avec intérêt $L$. Dumont, Essai sur l'individualisme: une perspective anthropologique de l'idéologie moderne, Edition du Seuil, Paris, 1983; G. Balandier, Structures traditionnelles et changement économique, in: Cahiers d'Etudes Africaines, $n^{\circ} 1,1960$.

Voir Ph. Haeriger, Stratégies privées, in: Enjeux fonciers en Afrique noire, Edition Khartala, 1982. 
d'établir le lien entre une population et une certaine morphologie ou une certaine culture. Les contacts entre les peuples et les civilisations au cours des siècles a favorisé un hybridisme morphologique et culturel qui a contribué à uniformiser les types d'hommes sans identité à $100 \%$ monoethnique. ${ }^{76}$ Un tel procédé est aussi dangereux dans la mesure où il institue un droit favorable au racisme et à la ségrégation, un modèle de droit qui a montré ses limites et qui a été rejeté sous d'autres cieux. ${ }^{77}$ On peut néanmoins envisager une solution plus raisonnable. Le législateur pourrait demander aux individus d'établir leur autochtonomie par la filiation. Il faudra simplement prouver que l'on est né de parents faisant partie d'une population autochtone.

Cette preuve est facile à établir pour les Camerounais nés avant 1972. Les actes de naissances établis par les officiers d'état civil jusqu'à cette date faisaient mention de la tribu ou de l'ethnie (population d'origine, en d'autres termes) des parents de l'enfant. ${ }^{78}$ Dans le dessein d'aboutir à l'intégration nationale en créant une communauté unique, toute référence tribale a été écartée dans l'établissement des actes d'état civil de naissance. Le rattachement d'un enfant à une population donnée est désormais difficile à établir, du moins sur le plan juridique.

Peut-être que le législateur reviendra sur cette mesure pour situer les enfants à naître dans leurs «populations» d'origine. Mais l'entreprise s'avère difficile une fois de plus, compte tenu des multiples brassages inter-ethniques déjà enregistrés; surtout lorsqu'on sais qu'aucun «fil d'Ariane» officiel permettant de remonter jusqu'à la tribu des ancêtres n'a été aménagé.

Comme on peut l'observer, la définition des populations ou des individus pouvant prétendre au bénéfice des dispositions constitutionnelles est loin d'être une affaire facile. Il faut néanmoins compter sur la sagacité du législateur qui trouvera sans doute une solution. Mais alors, une fois les définitions trouvées, d'autres difficultés se présenteront au législateur: la précision des droits fonciers à attribuer à ces autochtones.

77

Voir B. Toko, Alors qui est quoi, La Nouvelle Expression (déjà cité, note 42), p. 4.

Les lois sur la ségrégation raciale ont été vivement combattues dans le cas de l'Afrique du Sud. Elles ont été abolies aujourd'hui.

En réalité, on retrouvait sur les formulaires d'actes de naissance de l'époque la mention «race» qu'il fallait traduire par ethnie ou tribu. 


\section{B. Les difficultés liées à l'attribution des droits fonciers aux populations autoch- tones}

La réforme foncière du 6 juillet 1974 n'accorde aucun droit particulier aux autochtones. En vertu de la nouvelle Constitution, ce texte doit être repensé. Dans cette réforme qui se révèle incontournable aujourd'hui, il faut savoir que si l'espoir des autochtones peut être permis en ce qui concerne certaines terres susceptibles de leur être attribuées (2), on ne doit pas perdre de vue qu'il existe des terres qui sont, quoi qu'il en soit, insusceptibles d'être accordées aux autochtones (1).

\section{Les terres insusceptibles d'être accordées aux autochtones}

Une révision du texte foncier qui entend se faire respecter afin de ne point connaître de résistance doit se garder de porter atteinte à des droits déjà existants. Le domaine d'action du législateur dans une éventuelle réforme est alors limité. En accordant des droits aux autochtones, il sera difficile en tout cas de s'attaquer aux droits des autres particuliers et aux droits domaniaux.

\section{a) Les terres immatriculées}

On ne saurait, sous prétexte de redistribution des terres au Cameroun, s'attaquer aux terres immatriculées au profit des particuliers, terres qui font déjà l'objet d'un titre foncier. Certification officielle de la propriété immobilière, le titre foncier est selon l'article ler du décret du 27 Avril 1976, inattaquable, intangible et définitif. On ne saurait en tout cas, sous prétexte de redistribution des terres, balayer d'un revers de main le principe général de protection des droits acquis. Plus encore, une telle mesure porterait gravement atteinte à une autre disposition constitutionnelle, celle qui protège la propriété. ${ }^{79}$

Pourtant, il faut reconnaître que les autochtones gardent encore des prétentions sur ces terres immatriculées par des particuliers. Seulement, s'il faut revenir sur les droits de ces derniers pour satisfaire aux exigences des autochtones, on aboutirait à une expropriation pour cause d'utilité privée: une mesure qui tomberait elle aussi sous le coup de l'inconstitutionnalité.

79

«La propriété est le droit d'user, de jouir et de disposer des biens garantis à chacun par la loi. Nul ne saurait en être privé si ce n'est pour cause d'utilité publique et sous la condition d'une indemnité dont les modalités sont fixées par la loi», 24è paragraphe du Préambule de la Constitution du 18 janvier 1996. 


\section{b) Les terres du domaine public et privé de l'Etat}

Les populations autochtones ont souvent opposé des résistances au classement dans le domaine de l'Etat qu'elles considèrent comme appartenant à leurs ancêtres. Mais l'Etat a su imposer sa volonté par la force pour entrer en possession des terres. Les autochtones ont toujours fini par se résigner devant le tracé d'une route ou d'une voie ferrée, la construction d'un bâtiment administratif, l'installation d'un port maritime ou fluvial... L'incorporation des terres au domaine de l'Etat obeït à des besoins de développement. Mais elle se fait parfois au détriment de l'occupation effective des terres par les autochtones. Peut-on penser aujourd'hui qu'il s'agit d'accorder et de préserver les droits des autochtones, à une restitution de ces terres à ceux qui les ont toujours revendiquées?

Une telle opération serait politiquement impensable, socialement irréaliste et juridiquement critiquable. Sur le plan politique, une restitution des terres du domaine public et privé de l'Etat priverait l'Etat des infrastructures de fonctionnement, et risquerait de compromettre même son existence. En tant que personne morale, l'Etat a besoin de biens, et surtout des biens immobiliers. En effet, l'Etat doit être matérialisé par des installations à perpétuelle demeure, gage de sa perpétuité.

Sur le plan social, ce serait faire passer l'intérêt des particuliers que sont les autochtones avant l'intérêt général au service duquel sont placés les biens de l'Etat. Une mesure de restitution de ces biens immobiliers aux autochtones risquerait alors de mécontenter l'immense majorité des Camerounais et pourrait même entraîner des troubles sociaux. Il n'est pas exclu que la désapprobation vienne en premier des autochtones eux-mêmes. En effet, il a souvent été observé qu'après la réalisation des projets d'intérêt général sur leurs terres, les autochtones mécontents au départ finissent par apprécier le résultat qui leur profite aussi, et parfois en premier. Juridiquement, l'opération serait critiquable, en ce qui concerne les terres du domaine privé qui relèvent du droit de propriété. L'Etat en tant que sujet de droit mérite la même protection constitutionnelle que les particuliers en ce qui concerne son droit de propriété.

En ce qui concerne les terres du domaine public, elles sont inaliénables, imprescriptibles et insusceptibles d'appropriation privée. ${ }^{80} \mathrm{Ce}$ serait donc violer ces principes généraux attachés au domaine public que d'attribuer ces parcelles aux autochtones. Même lorsque les dépendances du domaine public sont reconnues sans utilité compte tenu de leur affectation

80

Article 2 alinéa 2 de l'Ordonnance $n^{0} 74 / 2$ du 6 juillet 1974. Voir J.M. Breton, La protection du domaine public, in: Encyclopédie Juridique de l'Afrique, Nouvelle Edition Africaine, 1982, p. 215 . 
initiale, elles ne peuvent être déclassées qu'au profit du domaine privé de l'Etat ou des autres personnes morales de droit public. ${ }^{81}$

Toutefois, certains biens classés aujourd'hui dans le domaine public pourraient exceptionnellement être restitués aux populations autochtones. Il faut penser ici aux concessions des chefferies traditionnelles et aux biens y afférents qui font aujourd'hui partie du domaine public de l'Etat. Ces concessions représentent des valeurs culturelles inestimables. On comprend alors le souci des pouvoirs publics qui entendent préserver ces valeurs au profit de toute la collectivité nationale et pourquoi pas au profit de la communauté internationale. C'est sans doute ce qui explique le classement de ces richesses dans le domaine public par la réforme de 1974.

Mais, qui mieux que les populations autochtones a conscience de ces valeurs culturelles? Qui mieux que ces populations pourrait sagement s'occuper du patrimoine des chefferies auxquels elles sont si solidement attachées? On pourrait donc sans risque quant à la préservation des richesses culturelles des chefferies, les restituer aux populations autochtones. Le rôle de l'Etat se limitera alors à un contrôle de gestion.

En dehors des concessions des chefferies traditionnelles, on comprend à travers les développements ci-dessus qu'il est impensable de dégager un espace foncier aux populations autochtones dans ce qui fait déjà partie du domaine public ou privé de l'Etat, encore moins dans les terres qui font dé jà l'objet d'une propriété privée. Il ne reste plus alors qu'un seul domaine où l'on peut rechercher un espace foncier aux populations autochtones: c'est le domaine national.

2. Les droits susceptibles d'être accordés aux populations autochtones sur le domaine national

Selon l'article 14 de l'Ordonnance de 1974, le domaine national est constitué des terres qui ne sont pas classées dans le domaine public ou privé de l'Etat ou des autres personnes morales de droit public; et des terres qui ne font pas l'objet d'un droit de propriété. L'article 15 de la même Ordonnance classe les terres du domaine national en deux catégories:

«1 - les terres d'habitation, les terres de culture, de plantation, de pâturage et de parcours donc l'occupation se traduit par une emprise évidente de l'homme sur la terre et une mise en valeur probante.

2 - les terres libres de cette occupation effective.»

81

Le principe est consacré par l'article 5 de l'Ordonnance $n^{\circ} 74 / 2$ du 6 juillet 1974, modifié par l'Ordonnance 77/2 du 10 janvier 1977. 
S'il est possible d'accorder des droits réels aux populations autochtones sur les terres de la première catégorie (b), on ne peut logiquement leur reconnaître que des droits personnels sur les terres de la seconde catégorie (a).

\section{a) Renforcement des droits personnels des populations autochtones sur les terres} libres de toute occupation effective

Les «terres libres de toute occupation effective» correspondent à ce que l'on a autrefois appelé «terres vacantes et sans maître». Il s'agit donc des terres que les autochtones ont toujours revendiquées. Aujourd'hui qu'il s'agit dans l'esprit de la Constitution de 1996, de faire droit à ces revendications, va-t-on assister à une restitution de ces terres aux populations autochtones? Peut-on penser à une transformation du domaine national en «domaine des populations autochtones»?

A première vue, l'entreprise semble périlleuse. En effet, s'il faut préserver les droits des populations autochtones comme l'exige la Constitution, il ne faut pour autant pas sacrifier les objectifs de développement. Dans un pays agricole comme le Cameroun, la terre reste une richesse essentielle sur laquelle comptent les acteurs du développement. Si l'on faisait des autochtones les maîtres de toutes les terres libres, il y aurait lieu de se demander si seules, ces populations seraient capables de les mettre effectivement en valeur. N'arriveraiton pas à une sorte de «thésaurisation» de cette richesse qu'est la terre? En tout cas, le Cameroun a besoin de forces autres que celles des autochtones, s'il entend réellement assurer son développement qui passe par le bon usage de la terre. Les agents du développement doivent trouver des terres disponibles; et cette disponibilité doit être sauvegardée par les pouvoirs publics. C'est pour cela qu'il est de bonne logique que les terres libres de toute occupation demeurent dans le domaine national géré par l'Etat.

Il est toutefois possible de concilier l'exigence constitutionnelle de préservation des droits des populations autochtones sur ces terres, avec les exigences de développement. Cette conciliation va consister en la reconnaissance des droits personnels aux autochtones sur ces terres. On pense ici à certains droits qui existent déjà au profit de tous les Camerounais sur le domaine national, et qui peuvent être simplement renforcés en faveur de ceux-là qui seront considérés comme autochtones dans un espace foncier.

L'article 17 de l'Ordonnance de 1974 accorde aux collectivités coutumières, à leurs membres ou à toute autre personne de nationalité camerounaise, un droit de chasse et de cueillette sur les terres du domaine national libre de toute occupation effective. L'exercice de ce droit par les allogènes a souvent donné lieu à des conflits; les autochtones ayant du mal à accepter qu'un «étranger» viennent faire de la chasse ou de la cueillette sur «leurs terres ancestrales». 
Pour préserver les droits des populations autochtones et mettre d'ailleurs fin aux multiples conflits entre celles-ci et les allogènes, on pourrait aménager aux autochtones un droit exclusif de culture de chasse et de cueillette sur les terres aux alentours de leurs villages, maisons d'habitation, plantations... On penserait bien à un rayon de quelques kilomètres ou toute autre dimension jugée utile. Les autochtones, par rapport aux espaces ainsi attribués, auront un droit exclusif de culture de chasse et de cueillette, tant que les pouvoirs publics n'auront pas donné à ces terres du domaine national, une affectation précise. C'est donc dire que l'Etat reste gestionnaire du domaine national. Il pourra tout à fait octroyer ces parcelles à des agents du développement économique. L'Etat pourra aussi, s'il le juge nécessaire, incorporer ces terres au domaine de l'Etat. Toutefois, les autochtones auront droit dans tous les cas, à une indemnité de perte de leur droit de culture de chasse et de cueillette. ${ }^{82}$

\section{b) Attribution des droits réels aux populations autochtones sur les terres qu'elles occupent ou exploitent effectivement}

Si les populations autochtones n'ont jamais trouvé la nécessité d'engager une procédure d'immatriculation sur les terres qu'elles occupent ou exploitent, c'est bien parce qu'elles s'y considèrent comme «propriétaires». Pourtant la réforme de 1974 ne leur reconnaît qu'un simple droit d'occupation et d'exploitation, c'est-à-dire en réalité, un droit qui est loin d'être un droit réel. On comprend alors que la situation des autochtones sur leurs prétendues terres est extrêmement précaire. Aujourd'hui qu'il s'agit de préserver les droits de ces populations il faut tout au moins leur reconnaître cette légitimité revendiquée depuis plus d'un siècle. Le problème qui se poserait alors est celui de savoir quels droits accorder aux autochtones sur ces terres. En tout cas, si l'on entend vraiment préserver leurs droits, il va sans dire que l'on devrait leur accorder des droits sans précarité.

On pourrait penser à un droit de propriété. Ceci se traduirait par une immatriculation d'office par les autochtones des terres en question. Mais seulement, en prenant une telle mesure, il faut encore se demander si l'on a avancé dans un sens favorable aux autochtones, puisque cette possibilité d'immatriculation avait déjà été offerte aux autochtones dans la réforme de 1974. Et le résultat est connu: les autochtones n'ont pas, ou n'ont même pas voulu profiter de cette opportunité. Il n'a jamais été question pour eux d'aller chercher une légitimité foncière en dehors de celle qu'ils tiennent de leurs ancêtres. Quoi qu'il en soit, on peut dire qu'il serait vain de demander une fois de plus aux autochtones d'immatriculer leurs terres, même si le formalisme procédural est réduit à sa plus simple expression. 
C'est l'Etat qui s'est engagé, à travers la Constitution, à préserver les droits des populations autochtones. Il doit donc aller jusqu'au bout de son engagement. En d'autres termes, il appartient à l'Etat de prendre les devants pour protéger les terres occupées ou exploitées par les autochtones. Plus concrètement, il s'agit simplement de renverser la procédure d'immatriculation: ce ne sera plus l'autochtone qui ira auprès de l'administration solliciter l'obtention d'un titre foncer; c'est plutôt l'Etat qui enverra son administration constater l'occupation ou l'exploitation des terres par les autochtones afin de leur délivrer des titres fonciers.

\section{Conclusion générale}

Une étude de la législation foncière camerounaise, quelques années après la Réforme de 1074 , montrait déjà la «nécessité pour l'Etat de repenser sa législation en la matière» ${ }^{83}$. En effet, plus que dans d'autres domaines du droit, l'ineffectivité de la loi de l'Etat est chronique en matière foncière. Et cela ne rassure personne. Au nom de la modernité, de l'intégration nationale et du développement économique, le législateur camerounais s'est toujours comporté comme si la réalité autochtone n'existait pas. La Constitution de 1996 a sans doute compris qu'en matière de droit, il faut compter sur les réalités du milieu. Elle pose les grands principes qui doivent être respectés par les législations. Seulement, on observe que plus de deux ans après la promulgation de la nouvelle Constitution, rien n'a encore bougé en matière foncière, comme dans d'autres branches du droit qui nécessitent aussi une toilette conforme aux exigences constitutionnelles: ainsi le texte législatif sur les regions qui doivent remplacer les provinces n'existe pas encore, la Cour constitutionnelle n'a pas encore été mise sur pied, le Sénat encore moins... En droit foncier comme ailleurs, il faut simplement être patient. Les réformes législatives sont attendues. Peut-être un texte foncier est-il déjà en préparation. Nous ne pouvons qu'espérer que les populations autochtones y trouvent leur compte. 
Africa's "artificial" boundaries: A colonial burden or a sensible solution?

\section{By Heinz Schneppen}

The boundaries established in colonial times are still the boundaries of most African states. Before independence, African leaders asked for a revision of these boundaries, which they considered arbitrary and artificial. When independence came, African leaders had changed their minds. A majority of African states opted for the preservation of the status quo, in agreement with international practice. A "revisionist" minority however ignored and challenged the doctrine of the OAU (which is to respect the boundaries as they were when the States achieved independence.)

There can be no doubt that the OAU-doctrine has contributed to the stabilization of states and boundaries in Africa. But events in Biafra, Eritrea, Sudan and Somalia have demonstrated that the principle of territorial integrity can conflict with the principle of selfdetermination (which is at least of equal importance).

In this context, secession might be considered a means of last resort, when selfdetermination is denied. But given the sometimes disastrous results of secession, states in Africa would be well advised to forestall the possibility of secession by granting internal self-determination (minority rights and autonomy) in time. The OAU would do well to abandon its rigid stance in favor of a more flexible position. African states should transform their organization, up to now lacking sanctions as well as resources, into an efficient (Organization for Security and Cooperation in Africa" (OSCA). Boundaries, in addition, would lose much of their devisive (and explosive) character through continental cooperation and regional integration. If African states could proceed in this way, the question as to whether their boundaries are artificial or reasonable would become rather academic.

The cameroonian land law in view of the constitutional regulations to protect the rights of autochtonous populations

\section{By Prosper Nkou Mvondo}

The new cameroonian Constitution of January 18, 1996, poses the principle of "protection of the rights of autochtonous populations". The autochtonous is defined in relation to land. The first right of an autochtonous population is then first of all, a right on his land of birth. By posing the principle, it stands to reason that the constitution calls for a land reform which will integrate the notion of autochtonous population, well known in the sociological domain, but ignored up to now by the cameroonian legal order in general and by the land 
law in particular. If the land regulation wants to be in accordance with the new constitution, which is a necessity, it must be reformed so as to grant and preserve the rights of the autochtones populations as the constitution requires it. In the socio-legal domain, the reform appears also as a necessity, given that the ineffectiveness of the present law of land is in connection with the resistances of autochtonous. The reform will then permit to satisfy their claims, in the interest of social peace and effectiveness of law. But to succeed, the lawmaker must take into consideration a number of legal problems: find a definition of the notion of "autochtonous population", find a space to grant them in a situation where all the lands have been already distributed. 